\title{
A REFORMA PSIQUIÁTRICA NA VISÃO DE QUEM CUIDA: PERCEPÇÕES DE PROFISSIONAIS DO SERVIÇO RESIDENCIAL TERAPÊUTICOa
}

\author{
Psychiatric reform in the vision of those who care: perceptions of professional of \\ therapeutic residential service \\ La reforma psiquiátrica en la visión de los que cuidan: percepciones de los profesionales \\ del servicio residencial terapéutico
}

\section{RESUMO}

O Serviço Residencial Terapêutico (SRT) tem se destacado como peça fundamental para a concretização do processo de desinstitucionalização e reabilitação psicossocial. Objetivou-se investigar as percepções dos profissionais de enfermagem atuantes no SRT sobre a Reforma Psiquiátrica, e sua relação com a formação técnico-científica e prática profissional. Estudo de natureza descritiva, com abordagem qualitativa, sendo entrevistados seis profissionais de enfermagem atuantes no SRT de Caicó-RN. A coleta foi realizada entre os meses de outubro e dezembro de 2009. A Reforma Psiquiátrica foi percebida como um movimento complexo que tem como finalidade modificar o trabalho na área da Psiquiatria; a partir dela o profissional é "motivado" a assumir uma nova posição em relação ao doente, uma postura mais horizontal e humanizada. Este estudo contribuiu para o melhor entendimento da realidade pesquisada, no que se refere à mudança na rede de saúde mental.

Palavras-chave: Equipe de Enfermagem. Prática Profissional. Saúde Mental. Serviços de Saúde Mental. Enfermagem Psiquiátrica.

\begin{abstract}
ABSTRACT

The Therapeutic Residential Services (TRS) has emerged as a key to the realization of the deinstitutionalization process and psychosocial rehabilitation. The objective was to investigate the perception of nursing professionals working in the TRS on the Psychiatric Reform, and your relationship with the technical and scientific training and professional practice. Descriptive study with a qualitative approach, being interviewed six nursing professionals working in TRS at Caicó/RN. The data collection was conducted between October and December 2009. The Psychiatric Reform was noticed as a complex movement that aims to modify the work in the field of psychiatry, and from it, the professional is "encouraged" to take a new position in relation to the patient, a more horizontal and humanized posture. This study contributed to a better understanding of the reality studied, regarding the change in the mental health network.
\end{abstract}

Keywords: Nursing, Team. Professional Practice. Mental Health. Mental Health Services. Psychiatric Nursing.

\section{Resumen}

El Servicio Residencial Terapéutico (SRT) se ha destacado como pieza fundamental para la realización de la desinstitucionalización y la rehabilitación psicosocial. El objetivo fue investigar la percepción de los profesionales de enfermería que trabajan en el SRT sobre la Reforma Psiquiátrica, y la relación con la formación técnica y científica y la práctica profesional. Estudio descriptivo con un enfoque cualitativo, mediante entrevistas con seis profesionales de enfermería que trabajan en SRT CaicóRN. Los datos fueran colectados entre octubre y diciembre de 2009. La Reforma Psiquiátrica fue notada como un movimiento complejo que tiene como objetivo modificar el trabajo en el campo de la psiquiatría, y de ella el profesional está "motivado" a tomar una nueva posición en relación al paciente, una postura más horizontal y humanizada. Este estudio contribuyó a una mejor comprensión de la realidad estudiada, en relación con el cambio en la red de salud mental.

Palabras-claves: Grupo de Enfermería. Práctica Profesional. Salud Mental. Servicios de Salud Mental. Enfermería Psiquiátrica.

${ }^{1}$ Bolsista PIBIC/UERN/CNPq. Acadêmica do Curso de Graduação em Enfermagem (Licenciatura e Bacharelado), Universidade do Estado do Rio Grande do Norte-UERN, Campus do Seridó, Caicó-RN, Brasil. E-mail: daniellerafson@hotmail.com.;²Enfermeiro. Mestre em Enfermagem (PGENF-UFRN), Doutorando em Ciências da Saúde (PPGCSa-UFRN). Professor Assistente II, Curso de Graduação em Enfermagem (UERN), Campus do Seridó, Caicó-RN, Brasil. Líder do Grupo de Pesquisa "A enfermagem no processo saúde-doença individual/coletiva, na educação em saúde e na assistência/gerência de serviços de saúde”. E-mail: professordulcian@gmail.com 


\section{INTRODUÇÃO}

A Reforma Psiquiátrica brasileira teve seu início ainda na década de 1970, sob influência de experiências internacionais de reversão efetiva do modelo hospitalocêntrico. Este movimento legitimou-se nas críticas ao saber psiquiátrico e à conduta médica, às práticas das instituições psiquiátricas e à negação dos direitos civis dos portadores de transtorno mental, objetivando a desinstitucionalização, isto é, a desconstrução do manicômio e de todos os paradigmas que o sustentam. ${ }^{1,2}$

Serviços de saúde mental substitutivos ao hospital foram criados com o intuito de garantir uma assistência contínua aos egressos, propondo novas formas de acolher/cuidar dos usuários segundo o paradigma da reabilitação psicossocial. Com isso, o modelo clínico e excludente que restringia o portador de transtorno mental ao asilo deu lugar ao modelo inclusivo clínico-epidemiológico, buscando devolver o doente à dinâmica da vida social e familiar, como forma de ressaltar suas potencialidades e vulnerabilidades no manejo com o seu adoecimento. ${ }^{3}$

Dentre os serviços substitutivos existem o Centro de Atenção Psicossocial (CAPS), o Serviço Residencial Terapêutico (SRT), os Serviços de Emergência em Psiquiatria, os HospitaisDia, entre outros. 0 SRT, com o propósito de reintegrar o portador de transtorno mental cronificado à sociedade, tem se destacado como peça fundamental para a concretização do processo de desinstitucionalização e reabilitação psicossocial, mediante significativa expansão e consolidação no território brasileiro.

Segundo dados do Ministério da Saúde, a série histórica dos SRTs em funcionamento no Brasil se dá de forma contínua e crescente, existindo, inicialmente no ano de 2002, 85 SRTs, e em 2010, 564 SRTs em funcionamento, acolhendo um total de 3.062 moradores. ${ }^{4}$

Estes serviços, criados para acolher os egressos (ou não) do hospital psiquiátrico, são casas localizadas no espaço urbano que devem apresentar um Projeto Terapêutico centrado nas necessidades dos moradores. Isso envolve mobilização dos profissionais de saúde e da comunidade na construção de estratégias de reabilitação, que primem pelo resgate da autonomia desses sujeitos e incentive/promova o exercício da

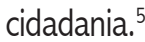

OSRT conta com o apoio/suporte clínico de uma rede de serviços de saúde mental, compreendidos pelo CAPS, Ambulatório de Saúde Mental, Hospital-dia e/ou a Estratégia Saúde da Família (ESF), a fim de garantir a assistência aos moradores.

A morada no SRT requer dos moradores a reinvenção de um cotidiano em uma casa, e é neste cotidiano que se busca aumentar a capacidade contratual, abrindo caminhos para reabilitação em um espaço de circulação e reconstrução de vínculos sociais e afetivos. Nessa perspectiva, a construção do cotidiano no SRT passa a ser um aliado na condução do tratamento desses moradores, entendida agora como uma clínica em movimento. ${ }^{6}$

Assim, ao ingressar no SRT, o morador dá início ao processo de reabilitação psicossocial com o reaprendizado das atividades cotidianas e interação no meio social/familiar, contando com o apoio do profissional cuidador (trabalhador de nível médio capacitado por uma equipe de referência) na condução da vida "extramuros" ao hospital. ${ }^{5}$

Com base neste princípio, oSRT está pautado no cuidado comunitário e humanitário, que, além de apresentar uma infraestrutura diferente do hospital psiquiátrico, direciona um novo olhar ao portador de transtorno mental, desviando da ênfase no tratamento dos sintomas (modelo de cunho biologicista) para enxergar o sujeito que sofre, que tem sentimentos, desejos, familia e amigos, e que, diante da patologia psiquiátrica, era desconsiderado. ${ }^{7}$

Este novo modelo de atenção proposto ao SRT e aos demais serviços substitutivos requer dos profissionais de saúde um elenco de estratégias de cuidado que contemple a integridade do indivíduo e sua família, e suas subjetividades, contrapondose às práticas de segregação e isolamento típicas do manicômio.

0 município de Caicó-RN vivencia uma transição na atenção a saúde mental, em que um passado recente de atrocidades ocorridas no único e extinto hospital psiquiátrico cedeu lugar, em 2008, a um SRT e um CAPS III (único do Estado).

Neste município, os cuidadores do SRT compreendem uma equipe de profissionais de enfermagem, que antes atuavam no hospital psiquiátrico local e que agora, junto à reestruturação dos serviços de saúde, são desafiados à superação de uma prática profissional vinculada ao controle, à vigilância e à punição, um processo de trabalho que, por anos, esteve fundamentado pelo saber da psiquiatria clássica e submetido à ordem médica.

Neste sentido, o presente estudo objetivou investigar as percepções dos profissionais de enfermagem atuantes no SRT sobre a Reforma Psiquiátrica, e sua relação com a formação técnico-científica e prática profissional.

É reconhecida a importância de novos olhares e ideias sobre os serviços de saúde mental de Caicó-RN, principalmente através da pesquisa científica bem direcionada. Sobretudo, fornecer subsídios para a reflexão do processo de Reforma Psiquiátrica vivenciado e orientação/conformação das práticas profissionais da equipe de enfermagem que atua no SRT. ${ }^{8}$

\section{METODOLOGIA}

Estudo de natureza descritiva, com abordagem qualitativa, desenvolvido no SRT pertencente à Secretaria Municipal de Saúde de Caicó-RN, entre os meses de outubro e dezembro de 2009. Participaram da pesquisa os profissionais de enfermagem atuantes neste serviço como cuidadores (três técnicos e dois auxiliares de enfermagem), além de um enfermeiro de referência, totalizando seis sujeitos de pesquisa. 
0 critério de inclusão estabelecido foi trabalhar há pelo menos um mês no SRT.

0 instrumento de coleta de dados utilizado foi a entrevista semiestruturada, contemplando a caracterização profissional (sexo, faixa etária, categoria profissional e experiência profissional na área de saúde mental) e o roteiro de entrevista propriamente dito. As questões norteadoras foram: 0 que você entende por Reforma Psiquiátrica? Que atividades você desempenha neste serviço de saúde mental (SRT)?

Os sujeitos foram esclarecidos sobre os objetivos e finalidades da pesquisa por meio da apresentação do Termo de Consentimento Livre e Esclarecido (TCLE). 0 projeto de pesquisa foi aprovado pelo Comitê de Ética em Pesquisa da Faculdade de Medicina do Juazeiro (CEP-FMJ, Parecer 2009/0543).

As entrevistas foram gravadas em áudio digital (aparelho MP4), e, posteriormente, transcritas na íntegra pelos pesquisadores. Os dados relativos à caracterização dos sujeitos foram apresentados em tabelas. Já os discursos sofreram a escuta flutuante e exaustiva, e foram agrupados segundo a semelhança de ideias, enquanto um artifício de análise.

A identificação dos entrevistados se apresenta pela sigla "Prof." (profissional), seguido pelo numeração de 1 a 6 , total de sujeitos entrevistados. Essa numeração foi dada de forma aleatória, dispensando a sequência das entrevistas e preservada a identidade dos sujeitos.

\section{RESULTADOS E DISCUSSÃO}

A partir do instrumento de pesquisa utilizado, os dados são apresentados e discutidos com subdivisão em dois tópicos: Caracterização dos Sujeitos da Pesquisa e Análise dos Discursos.

\section{Caracterização dos Sujeitos de Pesquisa}

Os sujeitos eram mulheres (100\%), casadas ( $83 \%)$, com idade superior a 30 anos, com mínima de 32 anos e máxima de 42 anos, inferindo possibilidade de experiência na área de saúde mental (Tabela 1).

Tabela 1 - Distribuição Absoluta e Percentual dos Sujeitos de Pesquisa segundo o sexo, estado civil e faixa etária. Serviço Residencial Terapêutico.

\begin{tabular}{lccccccccccccccccccc}
\hline & \multicolumn{1}{c}{ Sexo } & \multicolumn{1}{c}{ Estado Civil } & \multicolumn{1}{c}{ Faixa etária (anos) } \\
\hline \multicolumn{1}{r}{ Fem. } & Masc. & Total & \multicolumn{1}{c}{ Cas. } & \multicolumn{1}{c}{ Solt. } & \multicolumn{1}{c}{ Total } & $32-35$ & $36-40$ & $41-42$ & \multicolumn{1}{c}{ Total } \\
\hline $\mathrm{n}$ & $\%$ & $\mathrm{n}$ & $\%$ & $\mathrm{n}$ & $\%$ & $\mathrm{n}$ & $\%$ & $\mathrm{n}$ & $\%$ & $\mathrm{n}$ & $\%$ & $\mathrm{n}$ & $\%$ & $\mathrm{n}$ & $\%$ & $\mathrm{n}$ & $\%$ & $\mathrm{n}$ & $\%$ \\
\hline 6 & 100 & 0 & 0 & 6 & 100 & 5 & 83 & 1 & 17 & 6 & 100 & 2 & 33 & 3 & 50 & 1 & 17 & 6 & 100 \\
\hline
\end{tabular}

No tocante ao perfil profissional, a maioria era Técnico de Enfermagem (50\%) com tempo de trabalho na área de saúde mental/psiquiatria superior a 11 anos (67\%) (Tabela 2). Esse último dado revela que os entrevistados carregam a experiência do modelo asilar no seu currículo e prática profissional, o que pode se tornar um entrave, ou não, à incorporação dos pressupostos da Reforma Psiquiátrica, pois o processo de trabalho produzido nos hospitais psiquiátricos diverge do paradigma psicossocial reformista.

Sobre a atuação no SRT do Município de Caicó-RN, os entrevistados relataram um tempo de trabalho inferior a 1 ano (67\%), uma vez que sua implantação é recente na rede de serviços de saúde mental do município.

Tabela 2 - Distribuição Absoluta e percentual dos Profissionais segundo a categoria profissional, o tempo de trabalho na área da saúde mental/psiquiatria e no SRT de Caicó-RN em anos. Serviço Residencial Terapêutico.

\begin{tabular}{ccccccccccccccccccccccc}
\hline \multicolumn{1}{c}{ Categoria Profissional } & \multicolumn{1}{c}{$\begin{array}{c}\text { Tempo de trabalho na área de saúde } \\
\text { mental/ psiquiatria }\end{array}$} \\
\hline \multicolumn{11}{c}{ Aux. } & Téc. & Enf. & Total & $0-1$ & $2-10$ & $11-18$ & Total & $0-9$ & $10-16$ & Total \\
\hline $\mathrm{n}$ & $\%$ & $\mathrm{n}$ & $\%$ & $\mathrm{n}$ & $\%$ & $\mathrm{n}$ & $\%$ & $\mathrm{n}$ & $\%$ & $\mathrm{n}$ & $\%$ & $\mathrm{n}$ & $\%$ & $\mathrm{n}$ & $\%$ & $\mathrm{n}$ & $\%$ & $\mathrm{n}$ & $\%$ & $\mathrm{n}$ & $\%$ \\
\hline 2 & 33 & 3 & 50 & 1 & 17 & 6 & 100 & 2 & 33 & 0 & 0 & 4 & 67 & 6 & 100 & 4 & 67 & 2 & 33 & 6 & 100 \\
\hline
\end{tabular}

* Tempo em Meses

Em meio ao processo de mudanças, os profissionais de saúde mental são desafiados a se adequar a um novo campo de conhecimento, e a (re)construir novas práticas de saúde, elegendo-se a saúde mental e os novos serviços de tratamento (substitutivos) como espaços práticos de cuidados voltados ao processo saúde-doença mental do usuário e família.
No que se refere à formação, os profissionais cursaram disciplinas específicas sobre saúde mental/ psiquiatria durante a formação (100\%) e possuíam formação específica nesta área por meio de capacitações (67\%) e de eventos científicos na área.

Segundo alguns autores, a formação do enfermeiro ainda 
necessita de mudanças significativas para atender ao modelo psicossocial, principalmente no sentido de desconstruir os espaços burocráticos, controladores e excludentes que revigoram as práticas psiquiátricas. Um dos impedimentos para essa desconstrução seria as disciplinas de saúde mental/ psiquiatria oferecidas nos centros/escolas de formação, onde se prioriza o modelo clínico, baseado no ensino tradicional psiquiátrico, que resume a doença a um conjunto de sintomas que carecem de intervenção para resgatar o estado de normalidade. ${ }^{9,10}$

Trazendo esta análise para o âmbito do ensino técnico de Enfermagem, infere-se que os enfermeiros tendem a reproduzir o sistema de ensino pelo qual foram formados e, portanto, "alimentam" um ciclo vicioso de transmissão de saberes ultrapassados que pouco (ou nada) tem a contribuir para a atenção e reabilitação psicossocial.

Isso implica em dizer que as universidades, escolas e centros de formação em saúde devem reorientar o ensino no sentido de formar profissionais capazes de responder à nova tendência das práticas em saúde mental. Tal iniciativa possibilita transformações de saberes e práticas, para incorporar novas bases teórico-conceituais e técnicoassistenciais que respaldem um processo de trabalho imbricado pelos pressupostos da Reforma. ${ }^{9}$

No caso específico da proposta de formação do enfermeiro no Campus Caicó (UERN), o Projeto Pedagógico do Curso (PPC) estabelece que o conteúdo de saúde mental seja oferecido de forma transversal nas várias disciplinas específicas. ${ }^{11} \mathrm{~A}$ experiência de formação que se apresenta, ainda de forma inicial, parece demonstrar lacunas nos conteúdos de enfermagem em saúde mental/psiquiatria trabalhados nas disciplinas do currículo.

Com esta demanda, o curso adicionou uma disciplina optativa (Políticas Públicas de Saúde Mental), ofertada no semestre 2010.1, objetivando especialmente ressignificar a loucura enquanto fenômeno social e compreender o processo de Reforma Psiquiátrica vivenciado no Brasil. Essa iniciativa tem, a curto prazo, mostrado-se bastante relevante.

\section{Análise dos Discursos}

0 movimento de Reforma Psiquiátrica tem provocado na sociedade distintas interpretações que, por vezes, vão de encontro aos propósitos e diretrizes da Política Nacional de Saúde Mental, ou se afastam do seu entendimento, este último talvez por influência do antigo modelo manicomial.

Os múltiplos olhares sobre esse fenômeno são influenciados na medida em que as propostas reformistas, no âmbito teórico-conceitual, técnico-assistencial, político-jurídico e sócio-cultural, vêm ganhando visibilidade e destaque na sociedade. Além disso, os diferentes contextos em que seus atores (profissionais de saúde, usuários, familiares, gestores, comunidade) estão inseridos, também influenciam a complexa rede de interpretações acerca do transtorno mental e sua assistência requerida.
A Reforma Psiquiátrica foi percebida como um movimento complexo que tem como finalidade modificar o trabalho na área da Psiquiatria. 0 redirecionamento deste trabalho se daria no âmbito assistencial, fundamentado sobre o princípio da reinserção social dos portadores de transtorno mental, como ressaltado a seguir:

A reforma psiquiátrica é uma reforma de trabalhar o usuário [...]. Ela veio pra incluir o usuário à família, à sociedade, na qual se tem muito preconceito ainda. As pessoas tem! (Prof.4).

A reforma psiquiátrica éalgo quejá vem perdurando desde antes, de muito tempo. Na realidade em que nós trabalhamos aqui em Caicó, houve uma intervenção do Ministério da Saúde, passando o Hospital X a deixar de 'não existir' [...]. Então, se houver uma necessidade de uma internação pra eles [usuários], eles não são internados, eles são acolhidos (Prof.1).

Eu acho que essa reforma psiquiátrica veio mesmo pra [...] ajudar essas pessoas [portadoras de transtorno mental] [...]. Juntou ofísico com o social, para que eles voltem a viver na sociedade (Prof.2).

Este diferente enfoque dado ao trabalho em saúde mental não é uma tarefa fácil de ser compreendida, muito menos de ser realizada. No que se refere aos profissionais de saúde, a enfermagem de maneira especial apresenta maiores entraves, pois desde os primórdios da terapêutica manicomial foi influenciada pelo saber psiquiátrico e sua ideologia, pautando sua prática no isolamento, punição e repressão ao louco.

Desta forma, os estigmas de periculosidade e ameaça social, construídas historicamente sobre a loucura, e as suas formas de tratamento deixaram marcas nos trabalhadores da saúde e na sociedade, passando a ser modificados.

O que a gente vê é que os usuários hoje se envolvem melhor na sociedade. A sociedade hoje convive melhor com os usuários. As diferenças quando a gente trabalhava no Hospital $X$ e hoje CAPS, eu acho que são enormes. Antes eles [usuários] eram muito discriminados, hoje eu vejo mais todo mundo envolvido [...] tratando como se fosse gente normal mesmo, pessoas que se dizem normal como nós (Prof. 3).

Essa nova forma de acolher/assistir o portador de transtorno mental esteve, segundo os entrevistados, em consonância com a abertura dos novos serviços de saúde mental, na realidade do município de Caicó-RN. Isto é, a Reforma determinou a abertura de um novo serviço de saúde mental, 
que trouxe transformações diretas na assistência de enfermagem:

Não pode trancar eles, não pode conter, amarrálos, tem que deixar eles à vontade, sempre ocupando, interagindo com eles, fazendo acolhimento (Prof.1).

Eu, no meu plantão mesmo, deixo muito eles bem à vontade. "Olhe você tem que fazer isso", como no hospital [Psiquiátrico] [...] Ou "você tem que fazer aquilo". Eu não! Eu deixo eles bem a vontade, oriento e ajudo (Prof.4).

No tempo da psiquiatria, antes da reforma [...] ele [usuário] era o tempo todo trancado [...] a gente não via ele fora de maneira alguma. Aí, devido à reforma psiquiátrica, não pode mais trancar, não pode mais ter medo do paciente, tem que se aproximar (Prof. 6).

Como evidenciado, as práticas de "cuidado" antes realizadas no hospital psiquiátrico não podem mais ser executadas nos novos serviços de saúde mental, uma vez que se trata de propósitos e enfoques diferentes. Nesse sentido, 0 trabalho de enfermagem em saúde mental enfrenta uma transição entre a prática de cuidado hospitalar e a incorporação de princípios novos e desconhecidos que buscam superar a lógica manicomial. ${ }^{12}$

Entretanto, segundo os entrevistados, o trabalho de enfermagem não foi o único desafiado à superação. A sociedade como um todo foi levada também a refletir sobre a concepção da loucura e as consequências das práticas de cuidado produzidas. 0 discurso a seguir revela a visão do profissional a respeito do tratamento dado à loucura pela sociedade, e o motivo das mudanças pela Reforma.

\section{É trazer a humanização pra saúde mental. Porque antigamente, eles [usuários] eram tratados como bichos, a sociedade tratava como bichos. A sociedade e a familia também! Eu acho que eles [poder público] devido, muita denúncia que tinha antigamente, maus tratos, foram tendo um olhar mais humano diante desses pacientes (Prof. 6).}

A Reforma traz consigo um enfoque psicossocial sobre o transtorno mental, enxergando antes da doença o sujeito que sofre. E, a partir do momento em que se considera o doente um ser humano e cidadão, torna-se necessário também valorizar as influências psicossociais no seu processo de adoecimento. Nesse sentido, é responsabilidade da equipe interdisciplinar que atende nos serviços extra-hospitalares, a criação e manutenção de um ambiente terapêutico com a constante interação profissional-doente. ${ }^{13}$
Eu trabalho muito o lado o humano [...] não sou psicóloga, mas a gente dá assistência ao paciente, a gente orienta, eles confiam! Coloca a gente como referência, entendeu? E a função da gente não é dar o remédio, não é dar a injeção, é ter aquele contato, aquele pegar [...] todo ser humano necessita do toque, de atenção(Prof. 4).

A gente dá carinho. Está certo, tem as horas que a gente tem que falar sério também [...] Mas também a gente dá carinho, eles se apegam a gente. Eu acredito que tenha muita mudança nisso (Prof. 2).

Os depoimentos evidenciam que, a partir da Reforma, o profissional é "motivado" a assumir uma nova posição em relação ao doente, a adoção de uma postura mais horizontal e humanizada, que se propõe a desconstruir a imagem do profissional autoritário, tecnicista e impiedoso do modelo asilar. Essa nova posição é representada no SRT de Caicó, onde os profissionais tentam (re)criar um ambiente familiar.

Assim, não é pai e mãe, mas a gente faz com que fique um ambiente familiar, pelos menos da minha parte e das meninas [da equipe]. Até a gente não usa nem branco, a gente usa roupa normal pra tirar aquela imagem daquilo que era no hospital (Prof. 6).

Apesar de alguns depoimentos apontarem 0 entendimento e mudança de enfoque a partir da Reforma, existem, como ressaltado no início desta análise, inúmeras percepções sobre as ações desenvolvidas neste sentido, entre elas a não compreensão da necessidade/existência dos serviços substitutivos, em detrimento do fechamento do hospital psiquiátrico local.

Eles [poder público] tão vendo a Reforma, eu acho que é pra ver se ela melhora o lado da saúde mental [...]. E essa melhora que eles tão tentando fazer como tirar o hospital e botar o CAPS [...] eu não vejo melhora não (Prof. 5).

Esta opinião isolada de um dos membros da equipe de enfermagem do SRT talvez tenha respaldo no fato de que 0 SRT pesquisado não possui uma referência clínica definida, seja pelo CAPS existente (modalidade III) no município, ou ainda pela equipe da ESF do território. No entendimento do profissional de enfermagem, isto gera desassistência dos usuários egressos do antigo manicômio.

A valorização do manicômio enquanto único espaço de cura ainda pode estar atrelada ao saber de enfermagem adquirido durante a formação, em que os conhecimentos da psiquiatria tradicional com os velhos "referenciais teóricos" de 


\section{A Reforma Psiquiátrica na visão de quem cuida}

disciplina parecem ser o responsável por construir e produzir uma força de trabalho em saúde, que se distancia da compreensão da complexidade da assistência em saúde mental que a atual política de saúde mental requer. ${ }^{9}$

Tal achado repercute de maneira negativa tanto na visão do profissional que vivencia a transição do modelo de serviços de saúde mental quanto para a sociedade, que pode interpretar este fato isolado como um descompasso da Reforma. $\mathrm{Na}$ verdade, parece mais ressoar como uma ingerência ou negligência do serviço, ou ainda da irresponsabilidade do poder público no acompanhamento das políticas públicas de saúde, em especial as de saúde mental.

0 discurso a seguir resume o motivo da descrença da profissional em relação à mudança de lógica do serviço:

Eu acho que piorou aqui pra Caicó, porque o único suporte que tinha era esse hospital e acabaram [...]. O paciente fica ali [Caps III] uns dias, depois vai pra casa e fica rodando no meio da rua [...]. Quer dizer, se o hospital ainda existisse, ele não estava daquele jeito, ele estava lá, fazendo o tratamento dele, e não está no meio da rua (Prof. 5).

Essa percepção por parte de uma das cuidadoras pode emergir de sua experiência e duradoura vivência no hospital psiquiátrico, eleito por ela como único espaço capaz de oferecer "tratamento" e acolher os usuários não assistidos pela rede de saúde mental vigente.

Em meio a esse contexto, cabe ressaltar que a Reforma Psiquiátrica elege a inclusão/participação da família como meio de potencializar as propostas de reabilitação psicossocial e de desinstitucionalização. Intenta-se que, ao sair do manicômio, 0 portador de transtorno mental encontre no seio familiar e comunitário estratégias que proporcionem melhora na qualidade de vida, prevenção (de recidivas) e promoção à saúde mental. Essas metas seriam alcançadas, sobretudo, pelos profissionais de saúde, com a desmistificação do estigma social da loucura por meio das atividades de educação em saúde. 14,15

Enfatiza-se ainda a importância da atenção básica na rede de assistência à saúde mental, uma vez que é conferida à ESF o desenvolvimento de ações de caráter preventivo e promocional à saúde mental. Nesse âmbito, o trabalho multiprofissional e interdisciplinar deve dar suporte à grande parte dos problemas de saúde mental do território, dispensando, portanto, os excessivos números de encaminhamentos aos níveis especializados. ${ }^{16}$

No entanto, o que as diretrizes propõem e o que se observa no contexto pesquisado são duas realidades ainda distanciadas. No município de Caicó-RN, os motivos que ocasionam esta discrepância podem ser semelhantes aos resultados de outra pesquisa ${ }^{15}$ : o desconhecimento da Reforma Psiquiátrica e a falta de capacitação em saúde mental dos profissionais da ESF; a falta de condições para o atendimento de casos aos portadores de transtorno mental na ESF; a inexistência de uma rede em saúde mental, ou ainda a falta de entrosamento com serviços de saúde mental do território.

Reforça-se, nesse novo modelo de atenção, a organização de redes sociais de apoio destinadas aos portadores de transtorno mental, a exemplo das associações/organizações de usuários, familiares e técnicos, ou ainda, o for talecimento de atividades terapêuticas, no caso das Oficinas Terapêuticas (OTs).

As OTs apresentam-se como uma ferramenta imprescindível de ressocialização e inserção individual em grupos, pois objetiva o trabalho, o agir e o pensar coletivos, conferidos por uma lógica inerente ao paradigma psicossocial, que é respeitar a diversidade, a subjetividade e a capacidade de cada sujeito. ${ }^{17}$

Deste modo, a capacidade do portador de transtorno mental em retomar as atividades cotidianas é possível quando as iniciativas partem não somente do usuário, mas de quem cuida, de quem está ao seu redor, de quem torce por sua reabilitação, e este movimento cristaliza os ideais da Reforma.

Nos discursos a seguir, os profissionais entrevistados enfatizam a importância do envolvimento de todos, família, comunidade e profissionais de saúde, com os moradores do SRT, o que reflete uma mudança de comportamento:

Eu acho que todo mundo procura fazer o melhor pra ajudar eles [usuários]. [...] Tanto que tem uns que diziam que não iam [morar no SRT], que não iam gostar, e hoje não querem sair mais de jeito nenhum. Eu acho que eles são bem tratados, todo mundo trata muito bem eles (Prof. 3).

Na residência [SRT]; tem dois exemplos que a residência, a família e a sociedade próxima contribuem muito [...]. Porque aqui ele achou, se identificou, lá no fundo ele sabe que tem um lar, uma casa, uma cama, um quarto, uma roupa, uma família, que lá no hospital não tinha (Prof. 6).

Quando ele [usuário] estava no Hospital [psiquiátrico] a familia tinha maior dificuldade de recebê-lo, em ajudar. Ehoje ele já fica com a família no final de semana, a gente dá a medicação a ele, ele passa o final de semana e tem aquele contato com a familia que ele nunca teve (Prof. 4).

A morada no SRT possibilita ao usuário, egresso do manicômio ou não, uma maior aproximação e envolvimento com a comunidade. Os equipamentos sociais da cidade, quando articulados às redes de saúde mental, intensificam as estratégias de reabilitação psicossocial. E, mesmo que o serviço de saúde na atenção básica seja incipiente, a população não 
deve privar-se de suas (co)responsabilidades, depositando-as somente no serviço especializado. Ela precisa realizar um movimento interno/externo de acolhimento e socialização.

Desta forma, o processo de Reforma Psiquiátrica envolve mais que uma iniciativa político-econômica de criação de estratégias públicas de saúde, requer participação social, seja na sua elaboração ou mais ainda na sua concretização. Para isso, os profissionais da saúde, principalmente os atuantes na área de saúde mental, devem ser conhecedores destes propósitos, pois a partir da mudança de postura do profissional nos serviços substitutivos, uma nova relação com a loucura será difundida e afeiçoada à comunidade/sociedade.

\section{CONCLUSÕES}

Nesta pesquisa, a Reforma Psiquiátrica foi percebida pelos profissionais de enfermagem como um movimento que propõe mudanças na organização dos serviços de saúde mental, na sua postura e prática profissional, exigindo outras maneiras de cuidar e enxergar os portadores de transtorno mental. Um movimento que ultrapassa a relação usuário-profissional de saúde e envolve o âmbito familiar e social.

Essa nova relação incumbida pela Reforma está fundamentada na perspectiva da desconstrução do caráter de periculosidade estabelecido historicamente, e construção de uma nova prática baseada no paradigma da reabilitação psicossocial. Na realidade pesquisada, este caminho ocorreu com a abertura dos novos serviços, compreendidos por um SRT e um CAPS III.

A não compreensão da necessidade/existência dos serviços substitutivos, em detrimento do fechamento do hospital psiquiátrico local, entendido como único espaço de tratamento para os portadores de transtorno mental, também foi encontrada nesta pesquisa. Talvez, isto decorra da deficiente articulação entre o CAPS III, o SRT e a ESF do território, e da inexistência de matriciamento em saúde mental.

Foi unânime a incorporação de um enfoque psicossocial no trabalho, com a adoção de uma postura mais horizontal e humanizada que procura responsabilizar-se pela evolução, recuperação e reabilitação dos usuários.

A realização desta pesquisa possibilitou uma maior aproximação e admiração pelo processo de trabalho na área da saúde mental. A literatura específica requerida e explorada ao longo do estudo permitiu a visualização do panorama vivenciado pelos mais variados estados brasileiros, o que contribuiu para o melhor entendimento da realidade enfrentada no município de Caicó, no que se refere à mudança na rede de saúde mental.

Espera-se que os resultados aqui apresentados possam fomentar a discussão e reflexão sobre o processo de Reforma Psiquiátrica vivenciado pelo município, elegendo-se estratégias de superação. Além do mais, configura-se também em um momento de debate e de troca de experiências entre gestores, antigos e novos trabalhadores e a academia.

\section{REFERÊNCIAS}

1.Amarante P. Loucos pela vida: a trajetória da reforma psiquiátrica no Brasil. $2^{\mathrm{a}}$ ed. Rio de Janeiro: Fiocruz; 2009.

2.Gonçalves AM, Sena RR. A reforma psiquiátrica no Brasil: contextualização e reflexos sobre o cuidado com o doente mental na família. Rev Latino-am Enfermagem. [on- line] 2001; [citado 2010 mar 25]; 9(2): 48-55.

3.Azevedo DM. Estudo representacional da participação familiar nas atividades dos centros de atenção psicossocial no município de Natal-RN [dissertação]. Natal. Departamento de Enfermagem, Universidade Federal do Rio Grande do Norte; 2008. [citado 2010 jan 10]. Disponível em: http: / / www.pgenf.ufrn.br/arquivos/teses/ dissertacao_final_dulcian_pdf_pgenf.pdf

4.Ministério da Saúde (BR). Saúde mental em dados, 7. Brasília(DF); 2010.

5.Ministério da Saúde (BR). Residências terapêuticas: o que são, para que servem. Brasilia(DF); 2004.

6.Marcos CM. A reinvenção do cotidiano e a clínica possível nos "serviços residenciais terapêuticos". Psyche, São Paulo, 2004 jul/dez; 8(14): 17990.

7.Silva DS, Azevedo DM. Implantação e funcionamento dos serviços residenciais terapêuticos: um recorte do cenário nacional. Cogitare Enferm. No prelo 2011.

8.Azevedo DM, Silva DS. The nursing staff and new practices in mental health: the residential therapeutic service as background. Rev Enferm UFPE On Line [periódico on-line]. 2010; [citado 2010 jan 03]; 1 (4): 45053. Disponível em: http://www.ufpe.br/revistaenfermagem/index.php/ revista/article/view/718/493

9.Silva ATM. et al. Formação de enfermeiros na perspectiva da reforma psiquiátrica. Rev Bras Enferm. 2004 nov/dez; 57(6): 675-78.

10.Lucchese R, Barros S. A constituição de competências na formação e na prática do enfermeiro em saúde mental. Rev Esc Enferm USP. 2009; 43(1): 152-60.

11.Universidade do Estado do Rio Grande do Norte-UERN. Projeto Político do Curso de Enfermagem. Caicó(RN): Campus do Seridó; 2009.

12.Oliveira AGB, Alessi NP. 0 trabalho de enfermagem em saúde mental: contradições e potencialidades atuais. Rev Latino-am Enfermagem. 2003; [citado 2010 mar 25]; 11(3): 333-40.

13.Villela SC, Scatena MCM. A enfermagem e o cuidar na área de saúde mental. Rev Bras Enferm. 2004 nov/dez; 57(6): 738-41.

14.Azevedo DM, Miranda FAN; Gaudêncio MMP. Percepções de familiares sobre o portador de sofrimento psíquico institucionalizado. Esc Anna Nery. 2009; 13(3): 485-91. 
A Reforma Psiquiátrica na visão de quem cuida

15.Azevedo DM, Miranda FAN. A família e os serviços substitutivos em saúde mental: um recorte da produção bibliográfica nacional em enfermagem. Rev Enferm UFPE On Line [periódico on-line]. 2009 [citado 2009 nov 28]; 3(3): 93-8. Disponível em http://www.ufpe.br/ revistaenfermagem/index.php/enfermagem/article/view/257/295.

16.Nunes M, Jucá VJ, Valentim CPB. Ações de saúde mental no Programa Saúde da Familia: conûuências e dissonâncias das práticas com os princípios das reformas psiquiátrica e sanitária. Cad Saude Publica. 2007 out; 23(10): 2375-384.

17.Azevedo DM, Miranda FAN. Oficinas terapêuticas como instrumento de reabilitação psicossocial: percepção de familiares. Esc Anna Nery. No prelo 2011.

\section{NOTA}

${ }^{a}$ Recorte do Relatório de Pesquisa PIBIC/UERN/CNPq (vigência 20092010), intitulada: "A equipe de enfermagem e as novas práticas em saúde mental: conformação e implantação do Serviço Residencial Terapêutico no município de Caicó-RN". 